Letters to the Editor

\title{
The 40th Annual Meeting of The Japan Society for Analytical Chemistry
}

\author{
Yoshimasa NiHEI
}

Institute of Industrial Science, University of Tokyo,

Roppongi, Tokyo 106, Japan

The 40th Annual Meeting of The Japan Society for Analytical Chemistry (JSAC) was held at the Hiyoshi Campus of Keio University on November $21-23,1991$. The meeting was organized by a committee comprising members of the Kanto branch of JSAC, with Prof. Y. Nihei (Institute of Industrial Science, University of Tokyo) serving as the chairman.

The number of participants was $1750, c a .7 \%$ more than that attending the 39th Annual Meeting in Nagoya, and the largest ever registered for the Annual Meeting series of JSAC. There were 591 original papers, 30 invited papers as well as a Techno-review with 24 oral presentations.

This Annual Meeting contributed to the celebration of the 40th anniversary of The Japan Society for Analytical Chemistry. Memorial lectures were given by Professor G. H. Morrison and Professor B. L. Vallee, who were elected as the honorary members of JSAC in 1991. A panel discussion was also planned for younger researchers on the subject "how research in analytical chemistry should progress in the 21st century". A symposium titled "what kinds of education in analytical chemistry are derirable these days" was also held and attracted many attendees.

The contents of the general sessions included all fields of analytical chemistry: atomic spectroscopy, liquid and gas chromatography, analysis of new materials, radiochemical analysis, environmental and geochemical analysis, UV and visible absorption spectrometry, kinetic analysis, analysis of medicines and biomaterials, chemiluminescence and bioluminescence analysis, flowinjection analysis, chemical sensors, biosensors, electroanalysis, solvent extraction, ion exchange, preconcentration, separation, IR and Raman spectroscopy, NMR and ESR spectroscopy, fluorescence and phosphorescence analysis, photoacoustic analysis, sampling and sample treatment, organic microanalysis, mass spectrometry, functional materials for sensing, identification and separation, X-ray analysis, thermal analysis, and theoretical fundamentals.

A ceremony to confer several JSAC Awards, which included lectures by honorable recipients, was held at the auditorium of Keio University in the afternoon of November 22. Medals of Merit of JSAC were presented to 62 technicians. The award recipients and
Table 1 Award recipients and award addresses

a) The Japan Society for Analytical Chemistry Awards

Keiichiro Hozumı (Kyoto Pharmaceutical University)

"Studies on Developments of Analytical Techniques

Based on Microchemistry and Plasma Chemistry".

Yoshiyuki Kıso (Faculty of Engineering, Hiroshima University)

"Basic Study of Electrophoresis Utilizing Complex-

Forming Equilibria and Its Application".

Katsumi Goто (Faculty of Science, Toyama University)

"Application of Hydrophobic Ion-Associates in

Analytical Chemistry".

b) The Japan Society for Analytical Chemistry Awards for Technical Achievements

Tameyuki Amano (Shionogi Research Laboratories, Shionogi \& Co., Ltd.)

"Contribution to Drug Analysis by Fluorescence and Light Absorption Method".

Yasushi Nakamura (Analysis Research Center, Nippon Mining Co. Ltd.)

"Development and Standardization of Analytical Methods for High Purity Metals and Compound Semiconductors".

Takashi Nagatanı (Saitama Institute of Technology) "Contribution to Analytical Electron Microscopy by Means of Energy Dispersive X-Ray Microanalyzer".

c) The Japan Society for Analytical Chemistry Awards for Younger Researchers

Hitoshi Nohta (Faculty of Pharmaceutical Sciences, Kyushu University)

"Application of Arylaliphatic Diamines as Fluorogenic Reagents to the Determination of Bioactive Substances".

Chuzo Fuлмото (Materials Sciences, Toyohashi University of Technology)

"Studies on Structural Techniques Coupled with Liquid Chromatography".

Hidefumi SaKamoto (Nagoya Institute of Technology)

"Separation and Sensing of Alkali Metal Ions with Novel Crown Compounds". 
addresses are summarized in Table 1.

An exhibition of instruments and scientific publications was opened during the 40th Annual Meeting on the campus. Several committee meetings related to analytical chemistry were held on November 23.

In advance of the 40th Annual Meeting of JSAC, a press conference was held with fourteen newspapers and TV stations on the evening of November 18 at the city hall of Yokohama. Doctor Keiichiro Fuwa (the president of JSAC), Prof. Y. Nihei (the chairman of the executive committee of 40th Annual Meeting of JSAC) and members of the publicity service committee summarized the selected topics from the presentations during the meeting. Some of them were reported in detail in several newspapers, together with an article concerning the 40th Annual Meeting.

It was due to the joint efforts of the many persons concerned that the meeting proceeded with great success. Special thanks are also extended to the members of Keio University.

(Received February 1, 1992) 